\title{
Pulse Wave Velocity Measuring System using Virtual Instrumentation on Mobile Device
}

\author{
Răzvan A. Ciobotariu, Cristian Foşalău, Cristian Rotariu
}

\begin{abstract}
Virtual instrumentation is a concept that permits customizable modular software measurement and the development of the user-defined tools for control, process and visualization of data, creating versatile systems, using modular programming, intuitive and easy to use. In this paper we investigate a possibility of using virtual instrumentation in the development of two physiological parameters monitoring system, in order to assess a cardiovascular parameter, the Pulse Wave Velocity (PWV). We choose to monitor this parameter due to major incidence and impact of cardiovascular diseases (CVD).
\end{abstract}

Keywords - cardiovascular diseases, mobile devices, pulse wave velocity, patient monitoring, virtual instrumentation.

\section{INTRODUCTION}

$\mathbf{V}$ IRTUAL instrumentation is one of the major development resulted from the extensive use of computers, which offers the benefits of customizable software development of user-defined tools for control, process, and visualization of data, creating versatile systems, using modular programming, intuitive and easy to use. In this paper we present the development and implementation of virtual instrumentation software for medical application running on a mobile device.

According to recent studies and statistics, cardiovascular diseases (CVD) are among the most frequent cause of death. The total cost and the indirect mortality cost estimated for CVD are higher than for any other major diagnostic group [1].

Early diagnosis of CVD could prevent further

Manuscript received October 27, 2012. Manuscript accepted February 17, 2013. This work was realized with the support of POSDRU CUANTUMDOC "DOCTORAL STUDIES FOR EUROPEAN PERFORMANCES IN RESEARCH AND INOVATION" ID79407 project funded by the European Social Found and Romanian Government and the support of PERFORM-ERA "Postdoctoral Performance for Integration in the European Research Area" (ID-57649), financed by the European Social Fund and the Romanian Government.

R. A. Ciobotariu and C. Foşalău are with "Gheorghe Asachi”" Technical University of Iasi, Faculty of Electrical Engineering, Dimitrie Mangeron, no. 51-53, 700050 IASI, ROMÂNIA (phone: +40-232-237627; e-mail razvan.ciobotariu@yahoo.com).

C. Rotariu is with the "Gheorghe Asachi" Technical University of Iasi, Faculty of Automatic Control and Computer Engineering, Dimitrie. Mangeron no. 27, 700050 ROMANIA, (phone: +40232231343; e-mail: cr.rotariu@yahoo.com). complications, leading to improvement of patient's quality of life and, for long term, can decrease the costs of the medical system. One step further to this goal is the long term monitoring of the patients, in their usual habitat and during daily activities, without restricting their freedom of movement.

The studies in the health area have shown the major influence that arterial stiffness has on appearance and evolving of atherosclerosis [2], [3], rheumatoid arthritis [4], complications due to high blood pressure, development of myocardium and coronary diseases, affection of vascular bed of several organs, such as brain or kidney [5]. One of the noninvasive techniques of assessing the arterial stiffness is the measurement of Pulse Transit Time (PTT) / Pulse Wave Velocity (PWV) [6]. PTT is a technique that measures the time needed for a blood pressure pulse wave to cross a known section of the arterial tree, and the PWV represents the ratio between the length of the measured arterial segment and the elapsed time from the ventricular ejection until the pulse pressure reaches the distal measurement site. The delay of the propagation is due to elasticity of the blood vessels walls [7]. During blood ejection from the left ventricle, the sudden rise of blood pressure is absorbed by the elastic walls of the aorta, a pulse wave propagates along the aorta, transmitting their energy to the aortic wall. The energy stored in the artery walls is released after the cardiac contraction has finished, helping to blood propagation.

The Moens-Kortweg model (eq. 1) [8] states that PWV depends on the elasticity of the blood vessel wall, its thickness and diameter, and the density of blood: the stiffer the artery, the faster a pressure pulse is propagated through it [9].

$$
P W V=\frac{\text { distance }}{P T T}=\sqrt{\frac{E h}{\rho 2 r}}
$$

$E$ represents the Young's module of the blood vessel, $h$ is its thickness, $\rho$ is the density of blood, and $r$ is the radius of the blood vessel.

The major determinants of PWV are: age [3], - with age increases the stiffness of arterial walls by replacing the elastic fibers with collagen [10], hence, the pressure wave generated by left ventricle ejection travels along the arterial vascular tree more rapidly; blood pressure [11], [12] - high arterial 
blood pressure is associated with an increased arterial stiffness, which generates an increase of systolic pressure and decrease of diastolic pressure [13]; gender; heart rate because of frequency-dependant viscoelasticity of the arterial wall: increased heart rate leads to an increased arterial stiffness [14]; and sympathetic nervous system - sympathetic activation increases blood pressure, heart rate and smooth muscle cells tonus, hence, increases arterial stiffness [15].

\section{MATERIALS AND METHOD}

\section{A. Hardware development of the system}

There are many devices used for measuring the PTT and PWV, by different techniques, as example: measuring the pulse transit time from carotid to femoral artery [16], using pressure sensors; measuring the delay between the ECG Rwave and the arrival of the pulse at the radial artery [17], recorded by a brachial obtrusive cuff, or by performing ECGgated Doppler measurements. The inconvenient of the methods described above is that a few of them can be performed ambulatory and without the presence of a welltrained operator [18].

Our system for measuring the PWV is based on recording the ECG and photoplethysmographic (PPG) signals. This technique uses the R-wave extracted from the ECG signal as the beginning of the ventricular contraction, which is the start moment of the pulse wave, which travels from the heart to distal regions. The arrival moment of the pulse wave to the index of the left hand is determined from the PPG signal (Fig. 1).

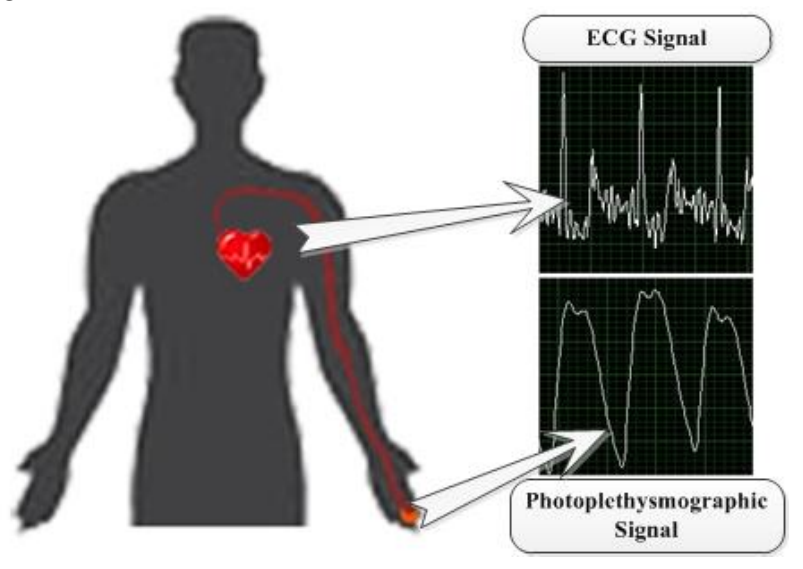

Fig. 1. Measurement sites of the ECG and PPG signals

The PWV was computed, as it is shown in equation (2), from the time difference between the starting point of the pulse wave and the moment when the pulse wave reaches the finger (Fig. 2).

By dividing the length of the blood vessel considered to the time measured from indexes difference, the PWV is determined.

$P W V=\frac{\text { blood vessel length } * \text { sampling frequency }}{\mid \text { Index }(E C G)-\operatorname{Index}(P P G) \mid}$
The length of the blood vessel was measured using anatomical landmarks for determining the heart position, the aortic branch and brachial trunk.

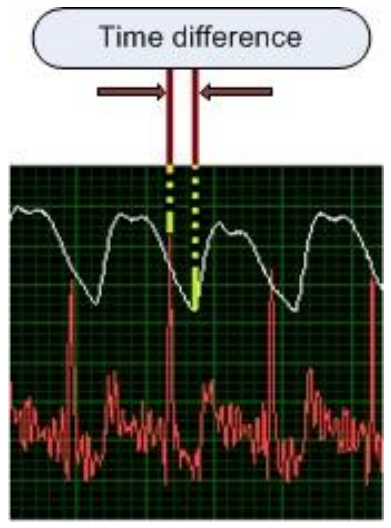

Fig. 2. Time difference between the ECG R-wave and the arrival moment of the pulse wave to the finger

The system was made using two ez430-RF2500 boards from Texas Instruments [19]. The ez430-RF2500 is an ultralow power wireless development system that has an MSP430F2274 microcontroller with a 10 bits A/D converter, for gathering and converting the data from the analog modules, and a wireless transceiver $\mathrm{CC} 2500$ at $2.4 \mathrm{GHz}$, working in ISM (industrial, scientific and medical) radio band, for data transmission. The ez430-RF2500 boards use for communication SimpliciTI [20], protocol developed by Texas Instruments and aimed at small RF ultra-low-power wireless sensor networks. The programming of the two ez430-RF2500 modules was done using the IAR Embedded Workbench for MSP430 [21].

One of the ez430-RF2500 modules was programmed to scan in the same cycle, both the ECG and PPG channels, at a frequency of 200 times per second, converting the signals from analog to digital.

The ECG module, made in our laboratory, is a low-power device, supplied from two AAA $1.5 \mathrm{~V}$ batteries and detects one lead ECG signal by measuring the skin potentials using 3 electrodes placed on the skin surface.

Fig. 3 presents the electrical diagram of the ECG module. The circuit amplifies the signal and filters it, first high-pass, rejecting the oscillations due to respiration, after that it filters low-pass, for rejecting the muscular activity noise.

The PPG module, also made in our laboratory, is powered from four AAA $1.5 \mathrm{~V}$ batteries, and uses a photodiode which emits light in IR domain. The IR fascicle is reflected by the finger skin, in accordance with the blood pulse wave which causes small oscillations of the light beam. A phototransistor detects these changes, causing a variation of its collector current. After that, a simple electronic circuit amplifies and filters the signal, creating the PPG wave. The electrical diagram is depicted in Fig. 4. 


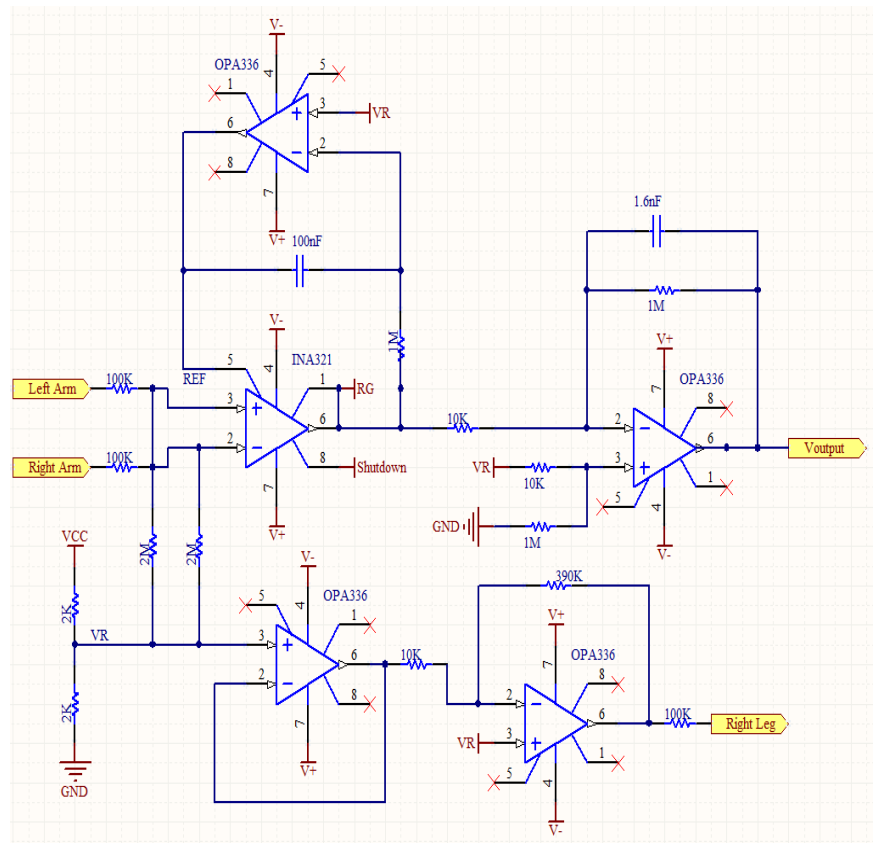

Fig. 3. ECG board schematic [22]

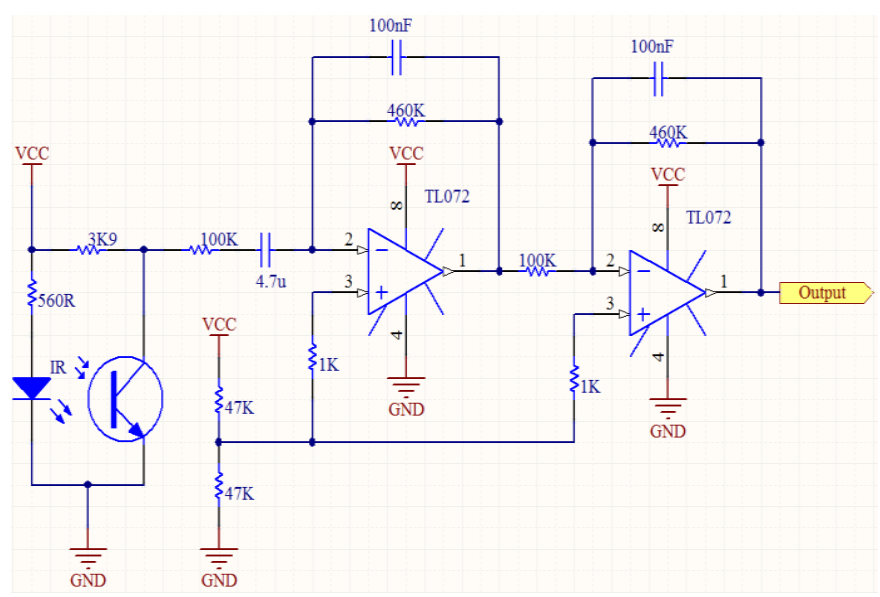

Fig. 4. PPG board schematic

After the A/D conversion, the ez430-RF2500 module packs the collected data and transmits them to the other ez430RF2500 module, which works as receiver, for saving them on the mobile device. Fig. 5 presents the ECG and PPG modules connected to the ez430-RF2500 transmitter module.

As mobile device, we choose a HTC X7500 smartphone, which has an USB interface, for connecting the receiver module, and uses Windows Mobile 5 as operating system (Fig. 6).

\section{B. Software development of the system}

The software that runs on the mobile device was developed by using LabVIEW 2010 from National Instruments [23], a graphical system design which offers the tools needed to create and deploy measurement and control systems.

To accomplish the signal processing, we developed and tested algorithms using Matlab 2011a software [24] to find

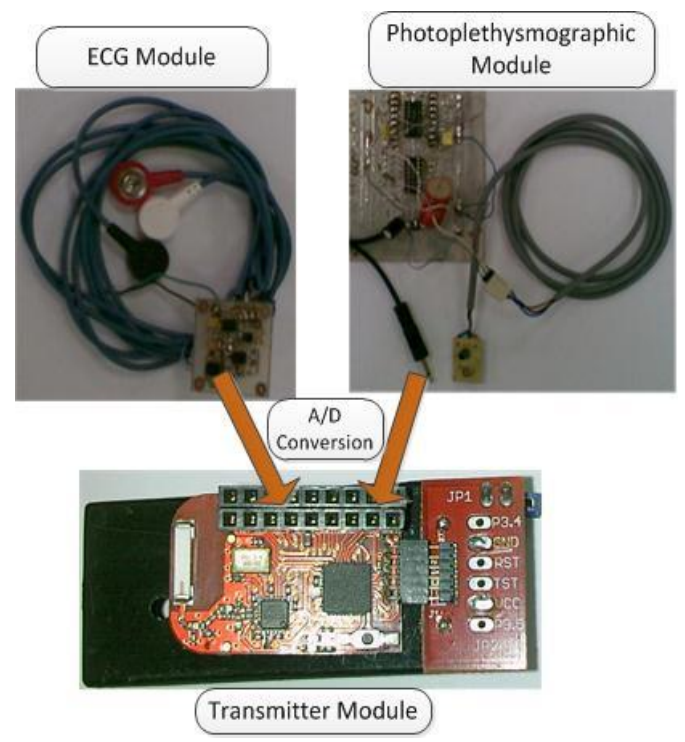

Fig. 5. ECG and PPG modules, connected to the transmitter module

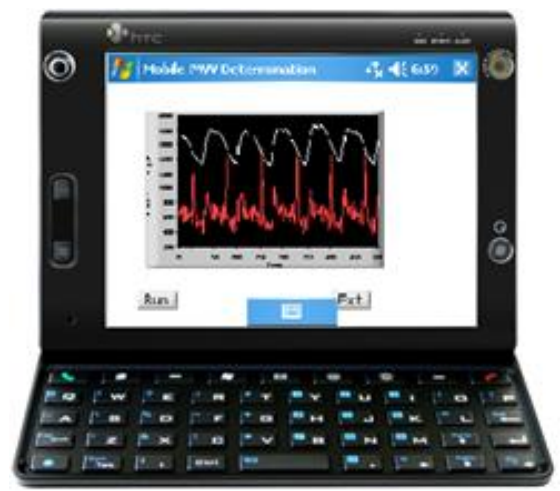

Fig. 6. HTC X7500 smartphone

the ECG R wave-indexes, to determine the PPG-indexes and to compute the PWV. After that, we implemented the algorithms on LabVIEW Mobile.

The software for PWV computation, running on the smarthphone as virtual instrument, is represented in the Fig 7.

The software unpacks the data as two separate signals and processes them separately.

For determining the ECG R-indexes and PPG-indexes, the algorithm uses a 500 points moving window.

First, the ECG signal is processed to determine the Rwaves and to extract the corresponding sample index. Because we were interested only on detection of the R-waves, which are the most prominent wave from ECG and the signal has a good signal to noise ratio, no additional filtration was necessary.

\section{RESULTS}

The constant used to compute the threshold was experimentally determined, and has the value around 0.5 .

The algorithm calculates the ECG threshold for each window, as follow in (3): 
Threshold $=\operatorname{mean}(\mathrm{ECG})+0.5 \times[\max (\mathrm{ECG})-\operatorname{mean}(\mathrm{ECG})]$

Using the equation (3), the algorithm finds the points that exceed the threshold and, in a 10 points window, it searches the maximum value, representing the maximum ECG R-wave point for that QRS complex. Finally, the window is moved and the whole process is restarted.

Fig. 8 presents in the top window, the found indexes on the ECG signal.

For finding the arrival moment of the pulse wave to the finger, the software follows the next steps:

Considering the ECG R-index previously determined to be the starting point for scanning the PPG signal, the software uses a 40 points moving window, in which searches the lowest signal value, considered to be the moment at which the pulse wave reaches the finger, and stores the value of that point and its index (Fig. 8. in bottom window).

The next step is to differentiate the indexes from the ECG R-wave and PPG and, multiplying the result with the sampling frequency, it is obtained the time passed from the start moment of the pulse wave until it reaches the finger (see (2)).

The final step on measuring the velocity of the pulse wave is to divide the knowing length of the arterial segment, to the time previously found (see (2)).

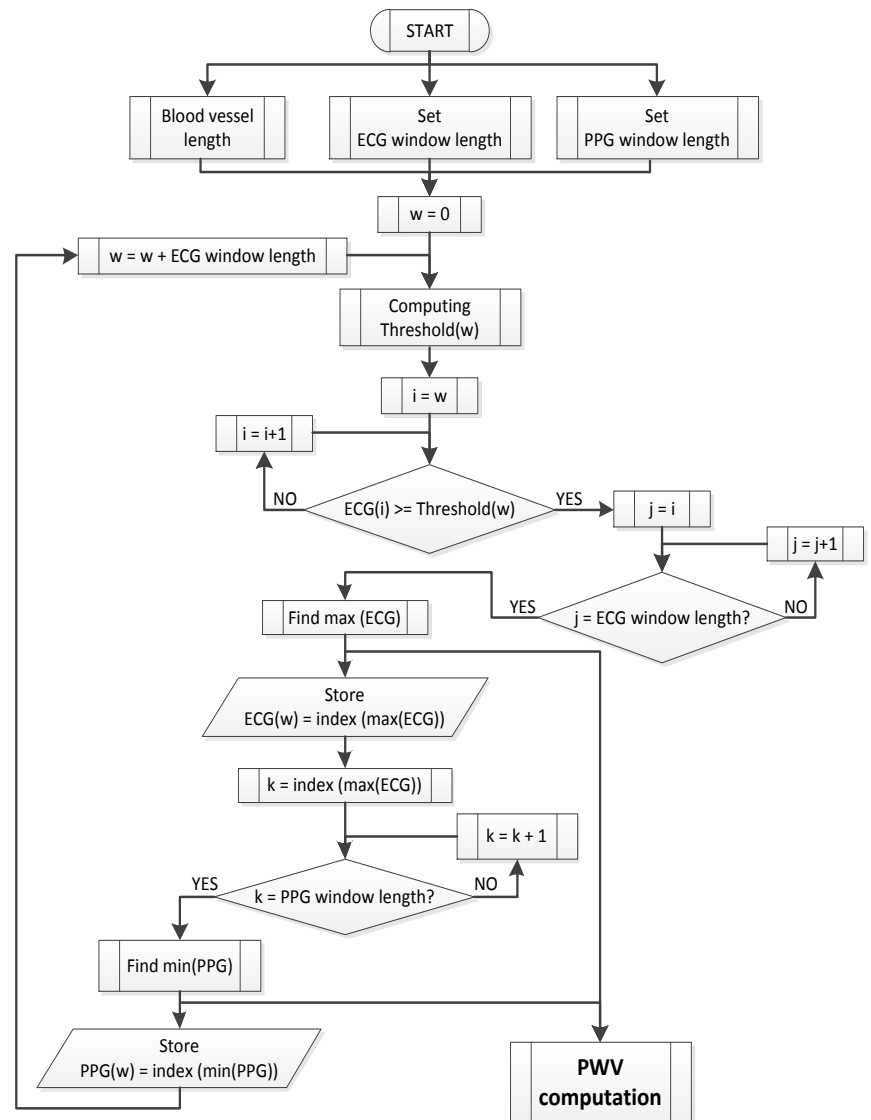

Fig. 7. Flowchart of the software running on LabVIEW for PWV computation

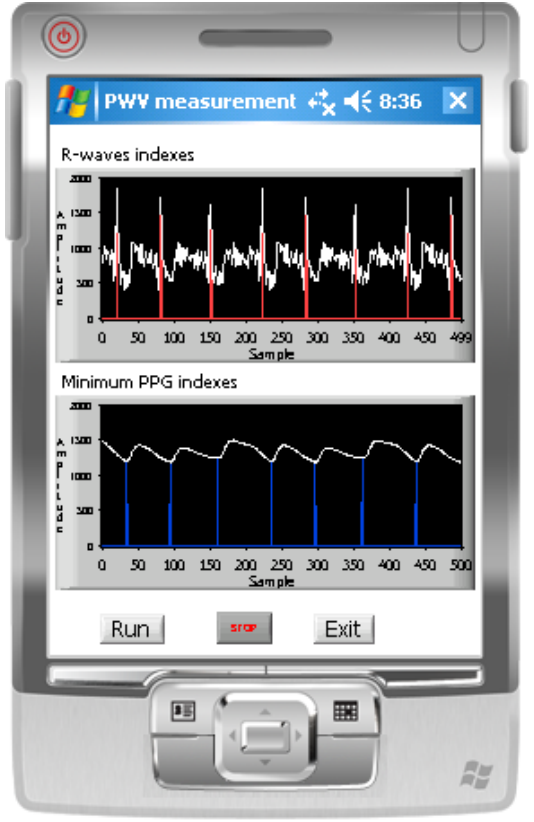

Fig. 8. LabVIEW interface for mobile device, displaying the ECG R and PPG indexes (screen shot on LabVIEW Windows Mobile 5 Pocket PC emulator)

After finding the values of PWV, the software creates a file and stores the data on the device.

The chart depicted in Fig. 9 represents the variation in time of the PWV recorded from a patient. The subject was resting and relaxing for a 15-20 minutes period of time and the PWV was recorded.

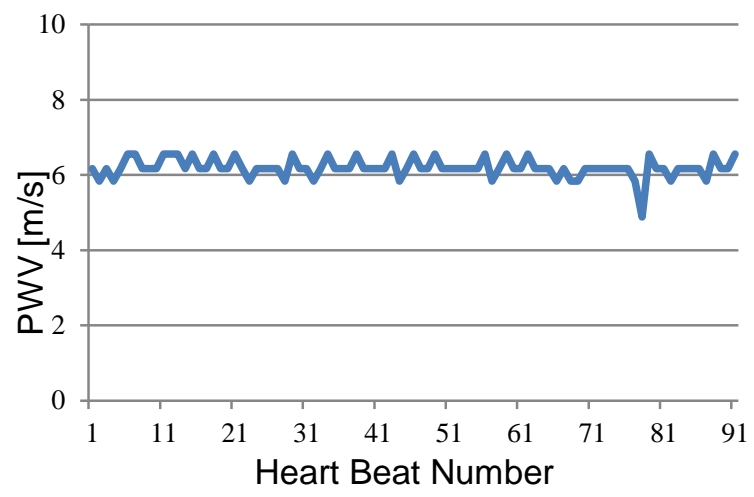

Fig. 9. PWV recorded while the subject was resting

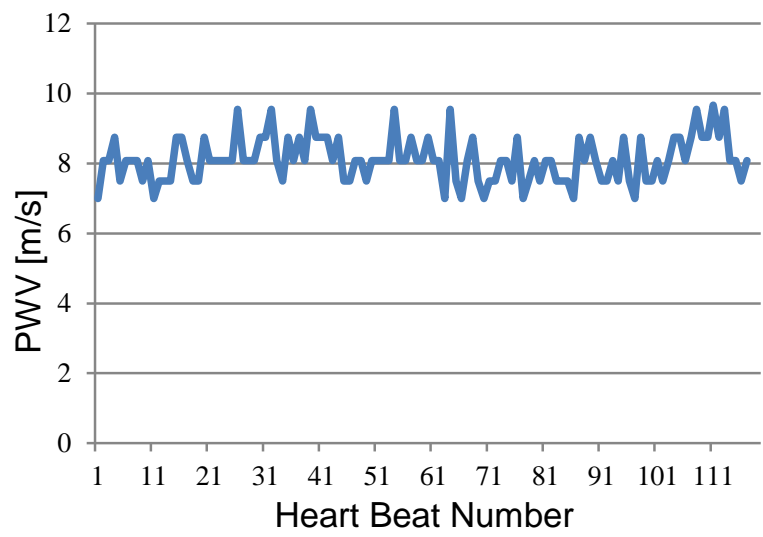

Fig. 10. PWV recorded after the subject had some physical effort 
The chart in Fig. 10 presents the variation in time of the PWV recorded from a subject after he had some physical effort, he rapidly climbed stairs for 10 minutes.

\section{CONCLUSIONS}

We choose to create a mobile medical device that perform long term PWV measurements, because this is an important parameter in determining the cardiovascular health or the response to some drugs administration, and we wanted to show the use of virtual instrumentation for developing devices with medical applications. Virtual instrumentation has the advantage of versatility, and due to graphical modular programming it is easy to learn and use, even by the untrained users.

In terms of medical applications and patient's welfare, we implemented the software on a mobile device, creating a tool for long term monitoring of patients at home, in their daily living and usual activities, which gives the freedom of movement, improving their quality of life. The mobile devices, such as PDA or smartphones, have the possibility to transmit data through GSM/GPRS or WiFi to the remote units, for the storage and further analysis. This represents one step forward in e-health and telemedicine.

\section{ACKNOWLEDGEMENT}

This work was realized with the support of POSDRU CUANTUMDOC "DOCTORAL STUDIES FOR EUROPEAN PERFORMANCES IN RESEARCH AND INOVATION" ID79407 project funded by the European Social Found and Romanian Government and the support of PERFORM-ERA "Postdoctoral Performance for Integration in the European Research Area" (ID-57649), financed by the European Social Fund and the Romanian Government.

\section{REFERENCES}

[1] http://circ.ahajournals.org/content/123/4/e18.full.pdf

[2] http://www.escardio.org/guidelines-surveys/escguidelines/GuidelinesDocuments/guidelines-AH-FT.pdf

[3] Hertzer N R 1991 The natural history of peripheral vascular disease. Implications for its management Circulation 83 (2 suppl.) I12-9

[4] "Arterial stiffness and central blood pressure, as determined by pulse wave analysis, in rheumatoid arthritis" R Klocke, J R Cockcroft, G J Taylor, I R Hall, D R Blake

[5] O'Rourke M.F., Safar M.E., "Relationship between aortic stiffening and microvascular disease in brain and kidney: cause and logic of therapy", Hypertension 46, 2005;

[6] Asmar R., "Arterial Stiffness and Pulse Wave Velocity. Clinical Applications", Elsevier, 1999;
[7] Pan Yi-Shan, Jia Xiao-Bo, Cui Chang-Kui, Xiao Xiao-Chun, Analyse of Pulse Wave of Pulse Wave Propagation in Arteries, Applied Mathematics and Mechanics (English Edition), 2006, 27(2):255-262.

[8] The Moens-Kortweg [x] model = Bramwell J. C. \& Hill A. V., "The Velocity of the Pulse Wave in Man", Proc. Royal Society for experim. Biology \& Med., Vol. 93, pp. 298-306, London, 1922

[9] Nichols W. W., Denardo S. J., Wilkinson I. B., McEniery C. M., Cockroft J., O'Rourke M.F., "Effects of Arterial Stiffness, Pulse Wave Velocity, and Wave Reflections on the Central Aortic Pressure Waveform", J Clin Hyoertens, 2008;

[10] Astrand H., Stalhand J., Karlsson J., Karlsson M., Sonesson B., Lanne T., "In vivo estimation of the contribution of elastin and collagen to the mechanical properties in the human abdominal aorta: effect of age and sex" J Appl Physiol 2011;

[11] B. Gribbin, A. Steptoe and P. Sleight, "Pulse Wave Velocity as a Measure of Blood Pressure Change", Psychophysiology, Vol.13, No.1, pp. 86-90, 1976.

[12] A. Steptoe, H. Smuylan and B. Gribbin, "Pulse Wave Velocity and Blood Pressure Change: Calibration and Applications", Psychophysiology, Vol.13, No.5, pp. 488-493, 1976.

[13] Asmar R., Beneros A., Topouchian J., Laurent P., Pannier B., Brisac A., Target R., Levy B., "Assessment of Arterial Distensibiliy by Automatic Pulse Wave Velocity Measurement", Hypertension, 1995;

[14] Benetos A., Adamopoulos C., Bureau J. M., et al. "Determinants of accelerated progression of arterial stiffness in normotensive subjects and in treated hypertensive subjects over a 6-year period", Circulation 105, 2002;

[15] Boutouyrie P., Lacolley P., Girerd X., Beck L., Safar M., Laurent S., "Sympathetic activation decreases medium-sized arterial compliance in humans" Am J Physiol, 267, 1994;

[16] Determination of age-related increases in large artery stiffness by digital pulse contour analysis S. C. MILLASSEAU, R. P. KELLY, J. M. RITTER and P. J. CHOWIENCZYK

[17] Non-Invasive Cuff-Less Measurements of the Arterial Blood Pressure: What does Pulse-Transit-Time tell us all about ? X. L. Aubert, J. Muehlsteff.

[18] Domenico Campolo, "New Developments in Biomedical Engineering”, ISBN 978-953-7619-57-2, InTech 2010

[19] http://www.ti.com/tool/ez430-rf2500

[20] http://www.ti.com/corp/docs/landing/simpliciTI/

[21] http://www.iar.com/en/Products/IAR-EmbeddedWorkbench/TI-MSP430/

[22] www.ti.com/lit/ds/symlink/ina321.pdf

[23] http://www.ni.com/labview/

[24] http://www.mathworks.com/ 